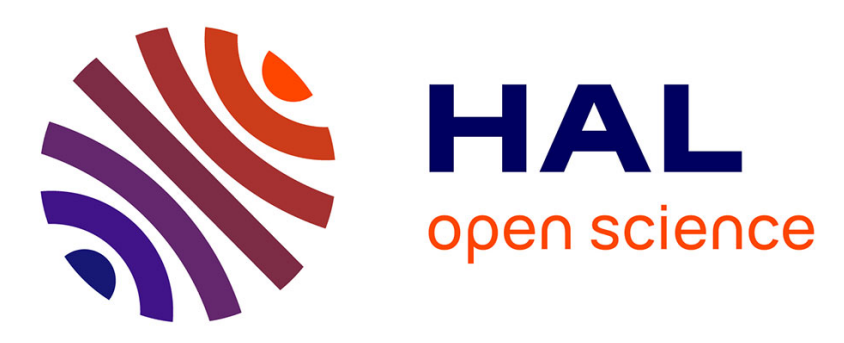

\title{
Architecture-Driven Level Set Optimization: From Clustering to Sub-pixel Image Segmentation
}

Souleymane Balla-Arabé, Xinbo Gao, Dominique Ginhac, Vincent Brost, Fan Yang

\section{- To cite this version:}

Souleymane Balla-Arabé, Xinbo Gao, Dominique Ginhac, Vincent Brost, Fan Yang. ArchitectureDriven Level Set Optimization: From Clustering to Sub-pixel Image Segmentation. IEEE Transactions on Cybernetics, 2016, 46 (12), pp.3181-3194. 10.1109/TCYB.2015.2499206 . hal-01430261

\section{HAL Id: hal-01430261 https://u-bourgogne.hal.science/hal-01430261}

Submitted on 9 Jan 2017

HAL is a multi-disciplinary open access archive for the deposit and dissemination of scientific research documents, whether they are published or not. The documents may come from teaching and research institutions in France or abroad, or from public or private research centers.
L'archive ouverte pluridisciplinaire HAL, est destinée au dépôt et à la diffusion de documents scientifiques de niveau recherche, publiés ou non, émanant des établissements d'enseignement et de recherche français ou étrangers, des laboratoires publics ou privés. 


\title{
Architecture-Driven Level Set Optimization: From Clustering to Sub-pixel Image Segmentation
}

\author{
Souleymane Balla-Arabé*, Member, IEEE, Xinbo Gao, Senior Member, IEEE, \\ Dominique Ginhac, Vincent Brost, Fan Yang
}

\begin{abstract}
Thanks to their effectiveness, Active contour models (ACMs) are of great interest for computer vision scientists. The level set methods (LSMs) refer to the class of geometric active contours. Comparing with the other ACMs, in addition to sub-pixel accuracy, it has the intrinsic ability to automatically handle topological changes. Nevertheless, the LSMs are computationally expensive. A solution for their time consumption problem can be hardware acceleration using some massively parallel devices such as graphics processing units (GPUs). But the question is: which accuracy can we reach while still maintaining an adequate algorithm to massively parallel architecture? In this work, we attempt to push back the compromise between, speed and accuracy, efficiency and effectiveness, to a higher level, comparing with state-of-the-art methods. To this end, we designed a novel architecture-aware hybrid CPU-GPU LSM for image segmentation. The initialization step, using the well-known k-means algorithm, is fast although executed on a CPU, while the evolution equation of the active contour is inherently local and therefore suitable for GPU-based acceleration. The incorporation of local statistics in the level set evolution allowed our model to detect new boundaries which are not extracted by the used clustering algorithm. Comparing with some cutting-edge LSMs, the introduced model is faster, more accurate, less subject to giving local minima, and therefore suitable for automatic systems. Furthermore, it allows two-phase clustering algorithms to benefit from the numerous LSM advantages such as the ability to achieve robust and sub-pixel accurate segmentation results with smooth and closed contours. Intensive experiments demonstrate, objectively and subjectively, the good performance of the introduced framework both in terms of speed and accuracy.
\end{abstract}

Index Terms - Level set method, image segmentation, graphics processing units, hybrid CPU-GPU architecture.

\section{INTRODUCTION}

$\mathrm{I}$ $\mathrm{N}$ computer vision and pattern recognition, image segmentation [1-7] is a major process by which a given image is partitioned into a number of meaningful and homogeneous regions, such that the union of any two neighborhood regions yields a heterogeneous segment. Due to the fact that there is no a general framework which is effective for all kinds of images, the task is non-trivial and more challenging in presence of noise. One should choose the proper method according to the characteristics of the image in hand.

In recent years, optimization methods have attracted much attention as powerful and natural image segmentation tools. Their basic principle is to achieve segmentation by minimizing

Corresponding author: S. Balla-Arabé is with the LE2I CNRS-UMR 6306, French National Center for Scientific Research, France (e-mail: balla_arabe_souleymane@ieee.org). a given energy function designed from the image information. Optimization methods can be roughly classified into two important classes: spatially discrete and spatially continuous representations. In spatially discrete approaches, the image pixels are usually considered as the nodes of a graph, and the aim of segmentation is to find cuts of this graph which have a minimal cost [8-9].

Active contour models belong to the spatially continuous approaches: the segmentation of the image plane is considered as a problem of infinite-dimensional optimization. The main idea is to evolve a given curve in the direction of negative energy gradient by means of an appropriate partial differential equation. The level set method (LSM) [10-13] designates the class of ACMs which uses the Eulerian framework, i.e., the geometric representation of the evolving curve, instead of the parametric one, i.e., the Lagrangian framework [14-17]. Fig. 1 displays the organization of the ACMs.

Comparing with parametric active contours, the LSM presents more advantages, such as the ability to easily handle complex shapes, and topological changes. Furthermore, it allows a straightforward passage from two-dimensional (2D) to 3D space. The original idea of the LSM stems from the Hamilton Jacobi approach, i.e., a time-dependent equation for a moving surface [18-20]. In space, the LSM aims to evolve a given curve toward its interior or exterior normal until defining the boundary of the object of interest. The curve evolution is driven by the level set equation (LSE) which is a nonlinear partial differential equation formulated as follow

$$
\frac{\partial \phi}{\partial t}=|\nabla \phi|(\mu V+\varepsilon k),
$$

where $\phi$ is the level set function (LSF), $V$ the velocity field which guides the active curve toward the needed boundaries, $\mu$ and $\varepsilon$ are user-controlling parameters and $k$ is a nonlinear curvature term expressed as

$$
k=\nabla \cdot \frac{\nabla \phi}{|\nabla \phi|}
$$

Two main approaches are usually used to stop the evolving curve on the contours of the desired object. The first uses an edge indicator depending on the gradient of the image, as in classical snake and active contour models [21-25]. These models have a lower computational complexity and are suitable to parallel architecture, but they are sensitive to noise and initialization. 


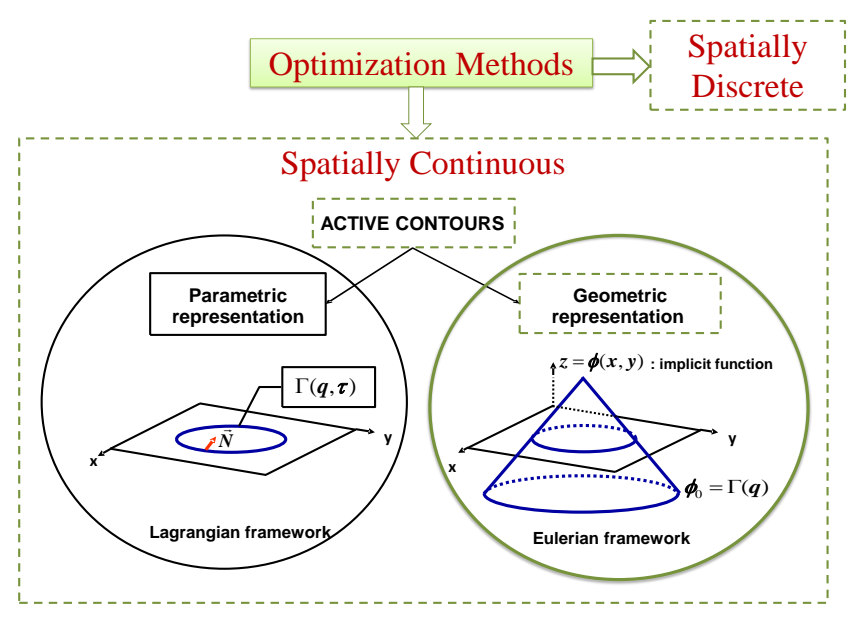

Fig. 1. Organization ACMs

The second uses some regional attributes to stop the evolving curve on the real boundary. These models are more robust against noise and can detect objects with weak edges. One of the most known region-based level set algorithms was proposed in [29] where Chan and Vese introduced a level set formulation to minimize the Mumford and Shah functional [30]. They converted the problem into a mean curvature flow problem just like the active contours. In [27], the authors used this approach in the field of remote sensing imagery for automatic detection of man-made objects (roads, buildings, etc.) from aerial and satellite images. In [52], Bazi et al. presented an unsupervised change detection method for multispectral images by designing a region-based energy functional using the difference image. The level set framework was then used to minimize the designed functional.

The region-based level set algorithms give better results than those of the classical active contours because the stopping term did not depend on the gradient of the image, reducing the dependence on strong edges and improving the robustness against noise. However, most of the times they cannot deal with intensity inhomogeneities, and are definitely not suitable for parallel programming, because they are not local. Furthermore, in order to solve the LSE, most of these methods suggest the use of some computationally expensive finite difference, finite element or finite volume approximations and an explicit computation of the curvature [31-32].

In our previous work [26] and [28], in order to handle the problem of computational expense, we used the lattice Boltzmann method (LBM) as an alternative approach for solving the LSE. Comparing with [29] for example, these approaches give better results in terms of efficiency and accuracy. The problem of time consumption is better handled because the curvature is implicitly computed and the algorithm is simple. But these introduced methods have some limits. In [26], we used regional attributes of a given pixel to design an Unsigned Pressure Force (UPF) which acted as a stop function for the active curve. This method is greatly dependent to the initialization which limits its use in automatic systems. Furthermore, the UPF is not local and therefore not suitable to parallel architecture-based acceleration. In [28], we used both local and regional statistics to design an energy function which had to be minimized using the level set framework, the method was more effective and robust against initialization than the one introduced in [26]. But as in [28], we still need to compute the mean values of pixels intensity inside and outside the active curve at each iteration, which increases the communication between the processors of any massively parallel device like GPU, and therefore slowdown the method.

In this work, we introduce a novel architecture-driven framework which allows obtaining a highly effective and fully local level set equation. Therefore, the proposed algorithm is adequate to parallel hardware-based acceleration, while allowing the achievement of promising segmentation results. In addition, the proposed method is a general framework which allows to considerably improving the results of most two-phase clustering methods such as k-means, the fuzzy c-means and their derivatives. By using the two intensity means output of the clustering algorithms and some local statistics of the image, we design an effective and inherently local energy functional. A fully local LSE is then obtained using the gradient descent methods. This strategy effectively allows combining, in a straightforward manner, the advantages of clustering technics and level set methods. On the one hand, the speed and the low complexity of some clustering algorithms, such as k-means, make the methods faster in comparison to some state of the art LSMs; on the other hand, the method has the advantages of the LSMs such as the ability to

1- achieve sub-pixel accuracy [29];

2- allow the incorporation of various prior knowledge, for example, shape and intensity distribution in order to achieve more robust segmentation results [33];

3- provide smooth and closed contours which are ineluctable for further applications such as shape analysis and recognition [34].

Although, most of two-phase clustering methods can be used, in this paper we use the output of the k-means algorithm, which has been initialized using the interior and the exterior intensity means of the initial active curve. The k-means algorithm, which is intrinsically fast and non-local, is executed on the CPU, while the level set evolution is executed on an NVIDIA GPU. The major contributions of the present work can be summarized as follows:

1- A novel inherently local and effective level set framework which perfectly suited to massively parallel architecture. The method can therefore be used in real-time computer vision systems;

2- A novel technic allowing some original clustering methods to benefit from the LSM's numerous advantages, and vice versa;

3- The introduction of local statistics in the level set evolution equation allowing the detection of new boundaries which are not extracted by the clustering algorithm. As a result

a. the minimization framework is less subject to giving local minima;

b. the framework is therefore less sensitive to initialization than the classical LSM and the k-means algorithm taken alone; 
c. the algorithm suits more to automatic systems;

4- A robust and effective hybrid CPU-GPU framework for fast image segmentation.

Intensive experiments, using the Berkeley segmentation dataset BSDS300 [35], demonstrate, subjectively and objectively, the effectiveness and efficiency of the proposed segmentation framework.

The remainder of the paper is organized as follows. Section 2 introduces the LBM. The formulation of the proposed method is given in Section 3. Section 4 presents the experimental results. The final section is the conclusion.

\section{BACKGROUND}

This section gives a general idea of the lattice Boltzmann model. The LBM was first designed to simulate Navier-Stokes equations for an incompressible fluid [37-39]. Its evolution equation is

$$
f_{i}\left(\vec{r}+\vec{e}_{i}, t+1\right)=f_{i}(\vec{r}, t)+\left(\frac{\partial f}{\partial t}\right)_{\text {coll }},
$$

which can be decomposed into two steps

$$
\begin{aligned}
& \text { Collision : } f^{\text {coll }}(\vec{r}, t)=f_{i}(\vec{r}, t)+\left(\frac{\partial f}{\partial t}\right)_{\text {coll }}, \\
& \text { Streaming: } f_{i}\left(\vec{r}+\vec{e}_{i}, t+1\right)=f^{\text {coll }}(\vec{r}, t),
\end{aligned}
$$

where $f_{i}$ is the particle distribution function and $\vec{r}$ a spatial variable. In this paper, $(\partial f / \partial t)_{\text {coll }}$ is the Bhatnager-GrossKrook (BGK) collision model [40-43], with a body force $\vec{F}$, expressed as

$$
\left(\frac{\partial f}{\partial t}\right)_{\text {coll }}=\frac{1}{\tau} \cdot\left[f_{i}^{e q}(\vec{r}, t)-f_{i}(\vec{r}, t)\right]+\frac{D}{b c^{2}} \cdot \vec{F} \cdot \vec{e}_{i},
$$

where $D$ is the grid dimension, $b$ the link number at each grid point, $c$ the length of each link which is set to 1 in this paper and $\tau$ represents the relaxation time. $f_{i}^{e q}$ is the local Maxwell-Boltzmann equilibrium particle distribution function expressed in its continuous form as

$$
f^{e q}=\rho(2 \pi R T)^{-3 / 2} \exp \left\{\frac{-(\vec{v}-\vec{u})^{2}}{2 R T}\right\}
$$

where $\vec{v}$ is the particle velocity and $\vec{u}$ the macroscopic velocity. The equilibrium distribution can be expressed in discrete form as follows when modeling typical diffusion phenomenon,

$$
f_{i}^{e q}(\rho)=\rho A_{i} \quad \text { with } \rho=\sum_{i} f_{i}
$$

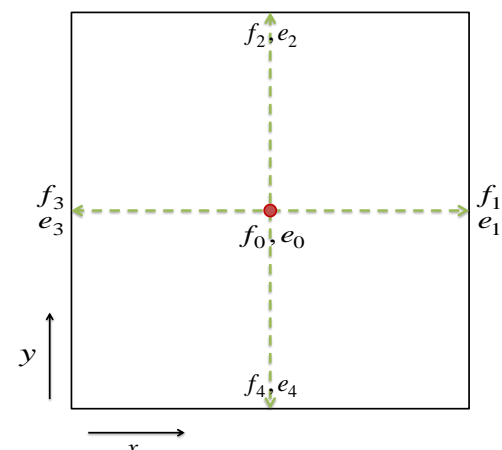

Fig. 2. Spatial structure of the D2Q5 LBM lattice

where $\rho$ is the macroscopic fluid density. By performing the Chapman-Enskog expansion [44], the following diffusion equation can be recovered from the lattice Boltzmann evolution equation [38],

$$
\frac{\partial \rho}{\partial t}=\beta \operatorname{div}(\nabla \rho)+F
$$

Substituting $\rho$ by the signed distance function $\phi$ in Eq. (9), the LSE can be recovered. In our model we use the D2Q5 ( $D=2, b=5$ ) LBM lattice structure. Fig. 2 displays a typical D2Q5 model where each link has its velocity vector $e_{i}(\vec{r}, t)$. The body force $F$ acts as the link with image data for the LBM solver.

Reference [45] used another approach to perform the level set image segmentation. Eq. (3) is the general evolution equation of the LBM; however in level-set-based image segmentation a stop function $g(\vec{r})$ is necessary to stop the evolving curve or surface at the boundaries of the object. In order to introduce the stop function into the LBM, the authors considered a medium between the nodes of the lattice. The particles can pass through the medium with a possibility of $g_{i}(\vec{r})$, and will be pushed back to where they were with a possibility of $1-g_{i}(\vec{r})$. The LBM evolution equation is modified as

$$
\begin{aligned}
f_{i}\left(\vec{r}+\vec{e}_{i}, t+1\right) & =g_{i}(\vec{r})\left[f_{i}(\vec{r}, t)+\frac{1}{\tau}\left[f_{i}^{e q}(\vec{r}, t)-f_{i}(\vec{r}, t)\right]+\sigma\right] \\
& +\left(1-g_{i}(\vec{r})\right) f_{i}\left(\vec{r}+\vec{e}_{i}, t\right)
\end{aligned}
$$

where $\sigma$ is the convection coefficient. The macroscopic fluid density $\rho$ is set as a signed distance function. More details about this approach can be found in [45].

\section{THE PROPOSED HYBRID LEVEL SET FRAMEWORK FOR TWO-PHASE IMAGE SEGMENTATION}

This section is dedicated to the conception and analysis of the proposed algorithm. We first design a novel energy functional, which will be minimized using the level set framework.

Let $\phi: \Omega \rightarrow \mathbb{R}$ be an LSF defined on a domain $\Omega$. In all our work $\phi$ is a signed distance function positive inside the zero level contour $\Gamma$ and positive outside. The introduced energy functional is defined as follow 


$$
E(\phi)=E_{\text {main }}(\phi)+E_{\text {Regularization }}(\phi)
$$

where $E_{\text {main }}(\phi)$ is the main energy term which contains the regional information of the image to be segmented, $E_{\text {Regularization }}(\phi)$ is the constraint on the smoothness and the length of the evolving contour.

By using the gradient descent method, the LSE can be recovered from the above defined energy function

$$
\frac{\partial \phi}{\partial t}=-\frac{\partial E}{\partial \phi}
$$

where $\partial E / \partial \phi$ is the Gâteaux derivative [46] of $\varepsilon$. According to Eq. (11), Eq. (12) is equivalent to the following evolution equation

$$
\frac{\partial \phi}{\partial t}=-\frac{\partial E_{\text {main }}}{\partial \phi}-\frac{\partial E_{\text {Regularization }}(\phi)}{\partial \phi}
$$

\section{A. Design of $E_{\text {main }}(\phi)$}

The main energy term $E_{\text {main }}(\phi)$ has been designed so that:

1- It is inherently local in order to suit to massively parallel architecture,

2- Its minimization yields to an effective and accurate image segmentation even in the presence of outliers.

$E_{\text {main }}(\phi)$ is therefore defined as

$$
\begin{aligned}
E_{\text {main }}(\phi) & =\gamma \int_{\Omega}\left(1-\exp \left\{-\left(I(x)-P_{1} / \omega_{1}\right)^{2}\right\}\right) H(\phi) d x \\
& +\lambda \int_{\Omega}\left(1-\exp \left\{-\left(I(x)-P_{2} / \omega_{2}\right)^{2}\right\}\right)(1-H(\phi)) d x \\
& +E_{\text {Outliers }}(\phi),
\end{aligned}
$$

Where $E_{\text {Outliers }}$ is the energy to be minimized in order to make the method robust against outliers, $H$ is the Heaviside function, $x$ a spatial variable, $\gamma$ and $\lambda$ are user controlling positive constants, $\omega_{1}$ and $\omega_{2}$ are important parameters which can lead, if wrongly chosen, to an over or under segmentation result. In this work, we used $\omega_{1}=\omega_{2}=1$. The values of $P_{1}$ and $P_{2}$ are obtained as follows. The interior and the exterior intensity average of the initial active curve are used to initialize a two-phase k-means algorithm, $P_{1}$ and $P_{2}$ are the mean values of the two final classes obtained. This part constitutes the serial part of our algorithm, and it is fast since computational efficiency is one of the main advantages of the k-means algorithm.

The derivative of $E_{\text {main }}(\phi)$ with respect to $\phi$ can therefore be formulated as

$$
\begin{aligned}
\frac{\partial E_{\text {main }}}{\partial \phi} & =\delta(\phi)\left[\gamma\left(1-\exp \left\{-\left(I(x)-P_{\text {in }} / \omega_{1}\right)^{2}\right\}\right)\right. \\
& \left.-\lambda\left(1-\exp \left\{-\left(I(x)-P_{\text {out }} / \omega_{2}\right)^{2}\right\}\right)\right]+\frac{\partial E_{\text {Outliers }}}{\partial \phi} .
\end{aligned}
$$

In [26], we had defined an efficient energy term based on a 2D gray-scale histogram which can make the LSM more robust by avoiding the evolving curve stopping at the pixels which are more likely to be outliers. This energy term was defined as follows

$$
\begin{aligned}
\mathcal{E}_{2 D-h i s t}(\phi) & =\int_{\Omega}\left(\alpha m_{1}+\beta m_{2}-I\right) \\
& . \exp \left\{\mu\left(\left|I-I_{\text {mean }}\right|-\eta\right)\right\} H(\phi) d x d y,
\end{aligned}
$$

where $I_{\text {mean }}$ represents the local average, $\alpha$ and $\beta$ are user-controlling parameters, $\eta$ and $\mu$ are positive constants, $m_{1}$ and $m_{2}$ are respectively the mean intensity values inside and outside the active contour and have to be computed at each iteration as follows

$$
\begin{gathered}
m_{1}=\int_{\Omega} I(x, y) \cdot H(\phi) d x d y / \int_{\Omega} H(\phi) d x d y, \\
m_{2}=\int_{\Omega} I(x, y) \cdot(1-H(\phi)) d x d y / \int_{\Omega}(1-H(\phi)) d x d y .
\end{gathered}
$$

In this work, inspired by the energy term defined in Eq. (16), we define $E_{\text {Outliers }}$ as follows

$$
\begin{aligned}
& E_{\text {Outliers }}(\phi)=A\left(P_{1}, P_{2}\right) \int_{\Omega} \exp \left\{\mu\left(\left|I-I_{\text {mean }}\right|-\eta\right)\right\} H(\phi) d x \\
& \text { with } A\left(P_{1}, P_{2}\right)=\gamma\left(1-\exp \left\{-\left(I(x)-P_{1} / \omega_{1}\right)^{2}\right\}\right) \\
& -\lambda\left(1-\exp \left\{-\left(I(x)-P_{2} / \omega_{2}\right)^{2}\right\}\right),
\end{aligned}
$$

A straightforward analysis of $E_{\text {Outliers }}$ shows that the effect of it minimization is to make the present method robust against outliers by avoiding the evolving curve stopping at pixels for which the intensity value is far different from their local intensity average, that is, high gradient isolate pixels. The derivative of $E_{\text {Outliers }}$ with respect to $\phi$ can therefore be written as follows

$$
\frac{\partial E_{\text {Outliers }}}{\partial \phi}=\left[A\left(P_{1}, P_{2}\right) \exp \left\{\mu\left(\left|I-I_{\text {mean }}\right|-\eta\right)\right\}\right] \delta(\phi),
$$

By adding this term in the main energy, we finally get the following equation

$$
\frac{\partial E_{\text {main }}}{\partial \phi}=\delta(\phi) A\left(P_{1}, P_{2}\right)\left[1+\exp \left\{\mu\left(\left|I-I_{\text {mean }}\right|-\eta\right)\right\}\right]
$$

\section{B. Design of $E_{\text {Regularization }}(\phi)$}

In traditional LSM, the regulation term used as a constraint on the area and the length of the active contour [29] is expressed as

$$
\begin{aligned}
E(\phi) & =E_{\text {Area }}(\phi)+E_{\text {Length }}(\phi) \\
& =\vartheta \int_{\Omega} H(\phi) d x+v \int_{\Omega}|\nabla H(\phi)| d x,
\end{aligned}
$$


where $\vartheta$ and $v$ are user controlling parameters. Its derivative with respect to $\phi$ is expressed as

$$
\begin{aligned}
\frac{\partial E}{\partial \phi} & =\frac{\partial E_{\text {Area }}}{\partial \phi}+\frac{\partial E_{\text {Length }}}{\partial \phi} \\
& =\vartheta \delta(\phi)-v \operatorname{div}(\nabla \phi /|\nabla \phi|) \delta(\phi) .
\end{aligned}
$$

In this paper, we introduced a local statistics-based constraint in the classical regularization term. Therefore, the modified regularization term is expressed as follows

$$
E_{\text {Regularization }}(\phi)=\vartheta \int_{\Omega} H(\phi) d x+v \int_{\Omega}|\nabla H(\phi)| d x+C(\phi)
$$

$$
\text { with } C(\phi)=\sigma \operatorname{sign}(\nabla I) H(\varepsilon-\exp \{\|\nabla I\|\}) \int_{\Omega}(1-H(\phi)) d x,
$$

where $\sigma$ and $\varepsilon$ are positive parameters. The term $C(\phi)$ is designed in order to attract the evolving curve towards the sectors of the image domain where the gradient is medium or high. Since the LSF $\phi$ is defined so that it is positive inside the zero level contour and positive outside. A straightforward analysis of $C(\phi)$ leads to the following conclusion,

$$
\begin{aligned}
& \text { 1) } \phi \geq 0 \Rightarrow C(\phi)=0, \\
& \text { 2) } \phi<0 \Rightarrow\left\{\begin{array}{l}
\text { if } \exp \{\|\nabla I\|\}<\varepsilon \Rightarrow C(\phi)=\sigma \operatorname{sign}(\nabla I) \\
\text { if } \exp \{\|\nabla I\|\}=\varepsilon \Rightarrow C(\phi)=0 .
\end{array}\right.
\end{aligned}
$$

We can therefore notice that when the curve is evolving, $C(\phi)$ is different from zero and has the sign of $\nabla I$ when the curve is on a pixel for which $\exp \{\|\nabla I\|\}<\varepsilon$. This makes the curve expands or contracts in order to evolve toward higher gradient pixels.

But since $E_{\text {main }}(\phi)$ is designed so that the evolving curve cannot stop on high gradient pixels, in order to make the proposed model robust against outliers, the designed regularization term will increase the ability to detect objects delineated by medium edges and weak edges depending on the threshold parameter $\varepsilon$. The derivative of the proposed regularization term with respect to $\phi$ can be written as follows

$$
\begin{aligned}
\frac{\partial E_{\text {Regularization }}}{\partial \phi} & =\delta(\phi)[\vartheta-v \operatorname{div}(\nabla \phi /|\nabla \phi|) \\
& -\sigma \operatorname{sign}(\nabla I) H(\varepsilon-\exp \{\|\nabla I\|\})] .
\end{aligned}
$$

Finally, we obtain the following LSE

$$
\begin{aligned}
& \frac{\partial \phi}{\partial t}=-\delta(\phi)\left(A\left(P_{1}, P_{2}\right)\left[1+\exp \left\{\mu\left(\left|I-I_{\text {mean }}\right|-\eta\right)\right\}\right]\right. \\
& +\vartheta-v \operatorname{div}(\nabla \phi /|\nabla \phi|)-\sigma \operatorname{sign}(\nabla I) H(\varepsilon-\exp \{\| \nabla I||\})) \\
& \text { with } A\left(P_{1}, P_{2}\right)=\gamma\left(1-\exp \left\{-\left(I(x)-P_{1} / \omega_{1}\right)^{2}\right\}\right) \\
& -\lambda\left(1-\exp \left\{-\left(I(x)-P_{2} / \omega_{2}\right)^{2}\right\}\right) .
\end{aligned}
$$

In order to extend the evolution to all the level set of $\phi$, the gradient projection method [47] allows us to replace $\delta(\phi)$ by $|\nabla \phi|$. To maintain the suitability to parallel programming of our model, we use the local LBM to solve the obtained LSE. By setting $\phi$ as a signed distance function, that is, $|\nabla \phi|=1$, and constraining it to stay like that, Eq. (26) can be written as follow

$$
\begin{aligned}
\frac{\partial \phi}{\partial t} & =-A\left(P_{1}, P_{2}\right)\left[1+\exp \left\{\mu\left(\left|I-I_{\text {mean }}\right|-\eta\right)\right\}\right] \\
& -\vartheta+\sigma \operatorname{sign}(\nabla I) H(\varepsilon-\exp \{\|\nabla I\|\})+v \operatorname{div}(\nabla \phi),
\end{aligned}
$$

which is similar to Eq. (9) with the body force expressed as

$$
\begin{gathered}
F=-A\left(P_{1}, P_{2}\right)\left[1+\exp \left\{\mu\left(\left|I-I_{\text {mean }}\right|-\eta\right)\right\}\right] \\
-\vartheta+\sigma \operatorname{sign}(\nabla I) H(\varepsilon-\exp \{|| \nabla I \mid\}) .
\end{gathered}
$$

The proposed LSE can therefore be solved using the following lattice Boltzmann evolution equation

$$
\begin{aligned}
f_{i}\left(\vec{r}+\vec{e}_{i}, t+1\right) & =f_{i}(\vec{r}, t)+(1 / \tau)\left[\bar{f}_{i}(\vec{r}, t)-f_{i}(\vec{r}, t)\right] \\
& +D / b c^{2}\left(-A\left(P_{1}, P_{2}\right)[1\right. \\
& \left.+\exp \left\{\mu\left(\left|I-I_{\text {mean }}\right|-\eta\right)\right\}\right]-\vartheta \\
& +\sigma \operatorname{sign}(\nabla I) H(\varepsilon-\exp \{\|\nabla I\|\})),
\end{aligned}
$$

without the necessity to explicitly calculate the computational expansive curvature term since it is implicitly handled by LBM.

Fig. 3 illustrates the flowchart of the proposed level set based algorithm, where we can clearly distinguish which part is executed on the CPU and which one on the GPU.

The principal implementation steps of the proposed method are as follows:

Input: $\quad$ Initial zero level contour $\phi$ (signed distance function), $\varepsilon, \eta, \gamma, \lambda, \mu, \vartheta$ and $\sigma$.

Output: The final zero LSF contours $\phi$.

\section{Algorithm Steps:}

1 Run the k-means algorithm with the interior and exterior means of the initial active curve as initial values.

$2 \quad$ Compute the body force $F$ with Eq. (28).

3 Resolve the LSE using LBM with Eq. (29).

4 Accumulate the $f_{i}(\vec{r}, t)$ values at each grid point with Eq. (8), which generates updated values of $\phi$.

5 Find the contours (zero level contours of the LSF).

6 If the algorithm has not converged, i.e., $\left\|\phi^{t+1}-\phi^{t}\right\|>10^{-5}$, go back to step 3 . 


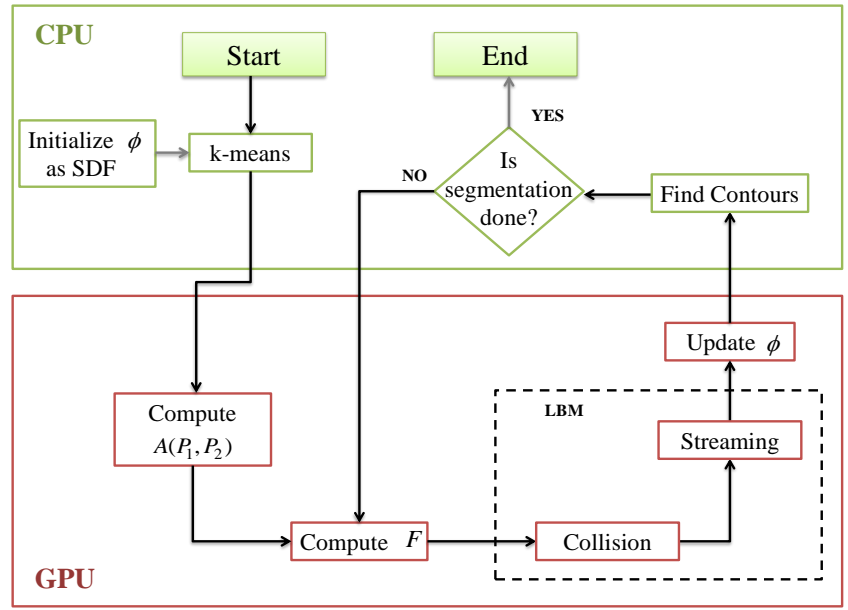

Fig. 3. Flowchart representing the process of the proposed algorithm.

\section{EXPERIMENTAL RESULTS AND ANALYSIS}

This section demonstrates the performance of the proposed architecture-driven level set image segmentation method (A-DLSO) both in terms of efficiency and effectiveness.

\section{A. Experimental setup}

The experiments environment is the parallel computing toolbox of Matlab R2013a installed on a laptop Aspire V3-571G with an Intel Core (TM) i3-2348M (2.3GHz, 3MB L3 cache) processor and possessing a GPU NVIDIA GeForce $710 \mathrm{M}$ with $2 \mathrm{~GB}$ dedicated VRAM. The executive time in all the experiments invoking the graphics card includes the transfer time of data from the CPU to the graphics card and vice versa. In this work, we have empirically set the parameters. In all the experiments, the chosen parameters are those who give the highest F-measure average. For our model, we fixed $\varepsilon=0.01, \eta=0.2, \gamma=5, \lambda=5, \mu=2, \vartheta=0$ and $\sigma=5$.

The optimized Matlab function arrayfun is used to execute the A-DLSO, the CV and the HZ code on the GPU. For example, the body force of the A-DLSO is computed using the following instructions

$$
\begin{array}{ll}
1- & \text { Id = gpuArray(I); } \\
\text { 2- } & \text { Imeand = gpuArray(Imean); } \\
\text { 3- } & \text { Fd = arrayfun(@Body_force,Id,Imeand }) .
\end{array}
$$

The first and the second instruction transfer respectively $I$ and $I_{\text {mean }}$ from the CPU to the GPU, while the third instruction computes the body force on the GPU using the kernel function Body_force.m programmed according to Eq. (28). Since all the output arguments are stored in the GPU memory, the function gather is used to transfer them back from GPU to CPU.

To best use the GPU capability, we restructure our code in order to maximally reduce the number of loops by using code vectorization technic. A good explanation of this method can be found in Matlab online support.

In this work, The GPU task and thread assignments are automatically handled by Matlab. Nevertheless, we should notice that the introduced model could be considerably faster by using the CUDA framework with our own task and thread management strategies. Furthermore, since the curve evolution is fully local, the use of CUDA kernels can provide a big speed-up, for example the collision step of the LBM can be a good candidate.

The supervised objective evaluation is undertaken using the F-measure based on precision and recall. It measures the similarity between two images. The higher it is, the better the segmentation result. It is formulated as follows [48-49]

$$
\begin{aligned}
& F=\left[\left(1+\rho^{2}\right) \cdot R \cdot P\right] /\left(\rho^{2} \cdot R+P\right) \\
& \text { with } R=\text { True Positive } /(\text { True Positive }+ \text { False Negative }) \\
& \text { and } P=\text { True Positive } /(\text { True Positive }+ \text { False Positive }),
\end{aligned}
$$

where $R$ and $P$ are, respectively, recall and precision, $\rho$ is usually set to 1 .

In order to better allow the subjective evaluation, we presented the results of all the methods that we used in two forms, by binary images and by only contours. To obtain the binary representation, the interior of the obtained contour is represented by white pixels and the exterior by black pixels. The blue triangle is the initial contour. The dimensions used are $450 \times 948$, with the exception of Fig. 4 where the dimensions of the image are of $948 \times 450$.

The proposed method and all the methods used for comparison were run using the intensity information $I$ of color images, which was obtained by performing a weighted sum of the R, G and B components as recommended by the 601 resolution of the International Commission on Illumination (CIE),

$$
I=0.2989 \times R+0.5870 \times G+0.1140 \times B .
$$

\section{B. Experimental results}

In this section we compare the introduced framework with the following methods

1- the recent parametric kernel graph cuts (PKGC) based image segmentation method introduced by Ben Salah and Mitiche in [50];

2- the region-based ACM (CV) introduced by Chan and Vese in [29];

3- the two-phase fuzzy c-means (FCM) clustering method;

4- the two-phase k-means clustering algorithm;

5- the local ACM (HZ) introduced by Hagan and Zhao in [51];

6- the ACM for image segmentation in the presence of intensity inhomogeneities introduced by Li et al. in [13];

7- the fast ACM (GF) described by Gibou and Fedkiw in [36].

Fig. 4 gives evidence of the way the proposed method improves clustering algorithms by allowing them to achieve sub-pixel accurate segmentation with smooth and closed contours. In this work, we used the mean values of the two final classes obtained by the k-means algorithm. Nevertheless, another clustering algorithm, such as the FCM or support vector machine (SVM), can be used. Fig. 6 (a) displays the original image with human segmentation as ground truth. As 
we can see from Figs. (b) and (c), more useful contours are detected by our model. In Fig. 6 (d), we superimposed the $\mathrm{k}$-means segmentation result with the final level set contour of the proposed algorithm represented in red. We can see that the contour is closed, smoother, and the F-measure, comparing the result with human segmentation, gives a score of $89.95 \%$ for our introduced framework and only $77.09 \%$ for the k-means. Thus, the result obtained by our model is far better in term of accuracy. This is an ineluctable proof on how it can greatly improve the quality performance of two-phase clustering algorithms.

Fig. 5 demonstrates the robustness of the proposed method against outliers. In this experiment, we simulated outliers using salt and pepper noise with different density values. The comparison with the k-means algorithm clearly shows the superiority of the proposed framework. The last row shows that even if the k-means fails, the A-DLSO is able to achieve a pretty good segmentation thanks to the use of local statistics, and the constraints on the area and the length introduced during the curve evolution.

From Fig. 6 to Fig. 8, we challenged the proposed algorithm using the PKGC, the CV, the FCM, the k-means, the $\mathrm{HZ}$, the $\mathrm{Li}$ and GF methods. The results of the supervised objective evaluation are displayed in Table I and the 3D histogram of Fig. 9. The executive times of the serial implementation on the CPU of all the algorithms used in this section are presented in Table II. Finally, the executive times of the GPU-based implementation of the A-DLSO, CV and $\mathrm{HZ}$ are displayed in Table III.

According to Table I, it can be seen that in almost all the experiments the proposed algorithm has the highest average F-measure, i.e., the produced result is the closest to human segmentation used as ground-truth. The subjective evaluation also confirms the superiority of the our model since it extracted more useful, thin and non-discontinuous contours. While the CV method got trapped in local minima, like in Fig. 6 (b), Fig. 6 (c) and Fig. 7 (b), the proposed method gives very good results which confirms its robustness against initialization, and therefore its suitability to automatic systems. Comparing with the A-DLSO, the HZ method presents lower performance, for example in Fig. 6 (a) and Fig. 8 (b), where it gives over-segmentation results. In many cases, the Li method also gives over segmented results, as demonstrated by Fig. 6 (a), Fig. 6 (c) and Fig. 8 (c). The GF method is less effective than the A-DLSO, particularly when it comes to detecting steep corners, or in terms of global segmentation like in Fig. 6 (b), Fig. 6 (c) and Fig. 7 (b). In comparison with the ground-truth, the A-DLSO gives better results than the parametric kernel based method, PKGC. As with the k-means clustering algorithm, the FCM and its derivatives can also be improved by our framework.

When comparing the executive times displayed by Tables II and III, we can see that even when serially implemented on the CPU, the proposed LSM is faster than almost all the methods used in these experiments. Aside from the k-means algorithm, only the $\mathrm{HZ}$ method, which uses a very simple speed function, is faster than the introduced method, but the quality of its results is far lower. The A-DLSO is far faster than the CV and the Li based LSM. In almost all cases, it is more than one hundred times faster than the $\mathrm{CV}$ method, and more than eighty times faster than the Li method. A straightforward analysis of the executive times of the GPU-based implementation evinces that the curve evolution part of the introduced method has been accelerated more than ten times compared to its serial version on CPU. This is a strong proof of its suitability to massively parallel architecture. The CV method which is non-local, because of the computation of the interior and exterior means of the active curve at each looping, has been accelerated only roughly two fold. Overall, we can conclude that the introduced A-DLSO presents very attractive performance in terms of speed comparing with level set based models.

Fig. 10 proves the robustness of the A-DLSO to initialization. It can be seen that from different initial contours, the proposed methods quietly produces the same finally good result, while the CV method is almost always trapped into a local minimum.

Table I. Statistical Results of the ObJective Evaluation Using F-MEASURE (\%).

\begin{tabular}{|l|c|c|}
\hline \hline Methods & Average & Standard deviation \\
\hline A-DLSO & 90.7841 & 0.9028 \\
\hline CV & 78.0608 & 2.7437 \\
\hline HZ & 76.1850 & 1.5957 \\
\hline k-means & 79.0566 & 1.0073 \\
\hline FCM & 79.9786 & 0.8641 \\
\hline PKGC & 83.1696 & 0.8743 \\
\hline Li & 81.7851 & 1.9599 \\
\hline GF & 78.1173 & 1.6935 \\
\hline \hline
\end{tabular}

\section{Conclusions And Perspectives}

This work presents an effective and efficient level set based image segmentation framework suitable for heterogeneous CPU-GPU architecture. The method which is also fast when serially implemented on the CPU, allows considerably improving the quality of the segmentation results obtained using most two-phase clustering algorithms such as SVM, k-means, FCM and their derivatives. Thus, by combining the advantage of LSMs and clustering methods, the A-DLSO is fast, gives smooth contours with sub-pixel accuracy, and can easily handle complex shapes. Its robustness to initialization makes it a good candidate for automatic systems. Intensive experiments demonstrate the good performance of the proposed method.

Future works will cover an extension of the A-DLSO to multiphase image segmentation, while conserving its intrinsic locality. The memory complexity aspect will also be considered, since it is one of the limits of the LBM used to solve the proposed LSE. Furthermore, some research on A-DLSO automatic parameterization will be carried out using some machine learning technics.

\section{REFERENCES}

[1] L. Chen, C. L. Philip Chen and M. Lu, "Multiple Kernel Fuzzy C-means based Image Segmentation," IEEE Transactions on Systems, Man, and Cybernetics, Part B: Cybernetics, vol.41, no.5, pp.1263-1274, 2011.

[2] H. Fu, X. Cao, Z. Tu and D. Lin, "Symmetry Constraint for Foreground Extraction," IEEE Transactions on Cybernetics, vol. 44, no. 5, pp. 644-654, 2014.

[3] S. Balla-Arabé, X.-B. Gao, and B. Wang, "A fast and robust level set 
method for image segmentation using fuzzy clustering and lattice Boltzmann method," IEEE Transactions on Systems, Man, and Cybernetics, Part B: Cybernetics, vol. 43, no. 3, pp. 910-920, 2013.

[4] C. Benedek, X. Descombes, and J. Zerubia, "Building Development Monitoring in Multitemporal Remotely Sensed Image Pairs with Stochastic Birth-Death Dynamics," IEEE Transactions on Pattern Analysis and Machine Intelligence, vol. 34, no. 1, pp. 33-50, 2012.
Simultaneous Image Segmentation and Bias Correction," IEEE Transactions on Cybernetics, vol. 45, no. 8, pp. 1426-1437, 2015.

[6] S. Balla-Arabé, and X-B. Gao, "Image multi-thresholding by combining the lattice Boltzmann model and a localized level set algorithm," Neurocomputing, vol. 93, pp. 106-114, 2012.

[5] K. Zhang, Q. Liu, H. Song and X. Li, "A Variational Approach to
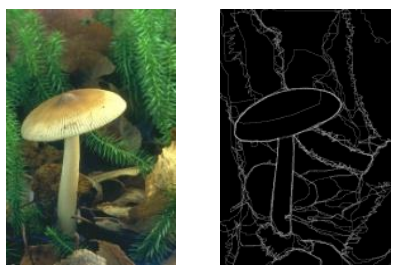

(a)

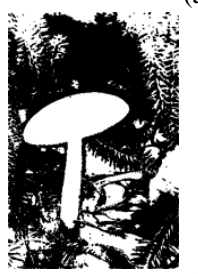

(b)
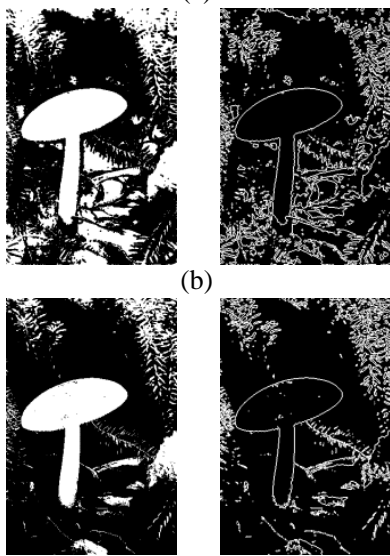

(c)

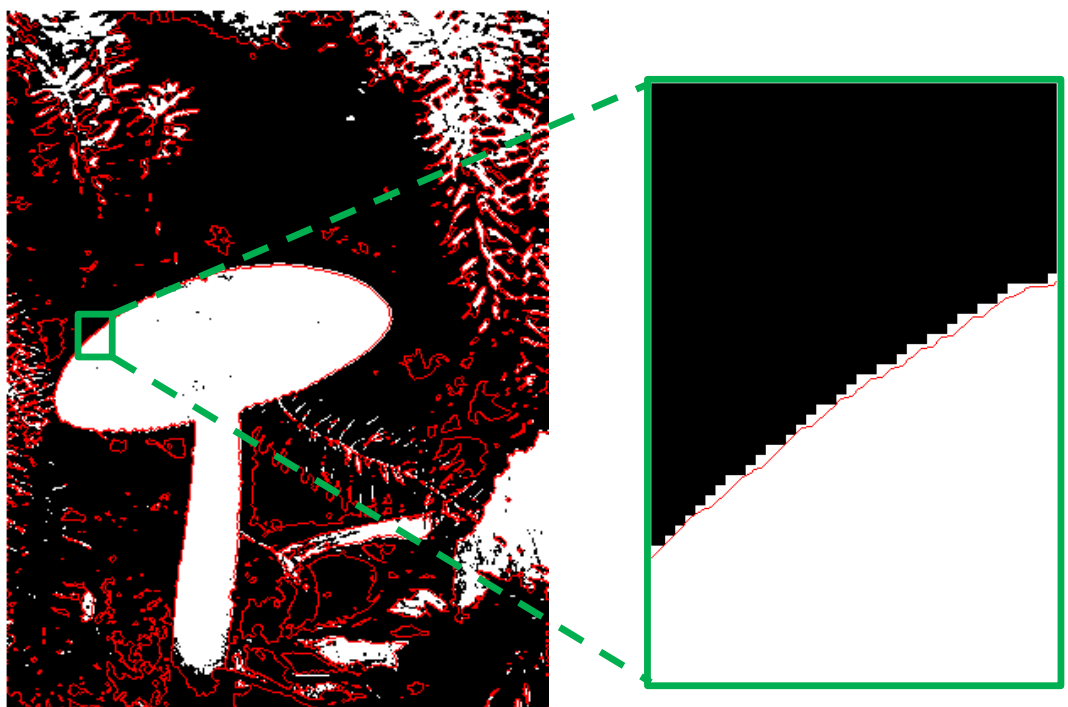

(d)

Fig. 4. (a) Natural image with human segmentation as ground truth. (b) Segmentation result of the proposed algorithm, binary and contour representations. (c) Segmentation result of the k-means algorithm, binary and contour representations. (d) Superimposition of the k-means result with the contours (in red) extracted by the method introduced in this paper.

Table II. EXecutive Times of THE SERIAL IMPLEMENTATION ON THE CPU (IN SECOND)

\begin{tabular}{|c|c|c|c|c|c|c|c|c|c|c|}
\hline \multirow{2}{*}{\multicolumn{2}{|c|}{ Methods }} & \multirow[t]{2}{*}{ Workspace } & \multicolumn{3}{|c|}{ Fig. 6} & \multicolumn{3}{|c|}{ Fig. 7} & \multicolumn{2}{|c|}{ Fig. 8} \\
\hline & & & (a) & (b) & (c) & (a) & (b) & (c) & (a) & (b) \\
\hline \multirow{3}{*}{ A-DLSO } & $\mathrm{k}$-means & \multirow{10}{*}{$\mathrm{CPU}$} & 0.6468 & 0.4394 & 0.4366 & 0.4706 & 0.4631 & 0.6523 & 0.5062 & 0.4733 \\
\hline & $\begin{array}{l}\text { Curve } \\
\text { evolution }\end{array}$ & & 0.2146 & 0.2107 & 0.2106 & 0.2092 & 0.2108 & 0.2160 & 0.2138 & 0.2122 \\
\hline & Total & & 0.8614 & 0.6501 & 0.6472 & 0.6798 & 0.6739 & 0.8683 & 0.7200 & 0.6855 \\
\hline \multicolumn{2}{|c|}{ CV } & & 85.007 & 84.520 & 85.694 & 84.971 & 84.066 & 85.443 & 137.55 & 84.741 \\
\hline \multicolumn{2}{|c|}{ HZ } & & 0.4497 & 0.4508 & 0.4595 & 0.4598 & 0.4603 & 0.4575 & 0.4562 & 04541 \\
\hline \multicolumn{2}{|c|}{ K-means } & & 0.6468 & 0.4394 & 0.4366 & 0.4706 & 0.4631 & 0.6523 & 0.5062 & 0.4733 \\
\hline \multicolumn{2}{|c|}{ FCM } & & 10.214 & 12.614 & 8.4572 & 10.355 & 9.3448 & 12.625 & 8.3827 & 12.758 \\
\hline \multicolumn{2}{|c|}{ PKGC } & & 2.6772 & 2.9213 & 2.4635 & 2.4909 & 2.4376 & 2.4485 & 2.7797 & 2.9501 \\
\hline \multicolumn{2}{|c|}{$\mathbf{L i}$} & & 70.2741 & 66.8958 & 74.2536 & 50.4556 & 55.4759 & 48.6515 & 79.9054 & 56.3074 \\
\hline \multicolumn{2}{|c|}{ GF } & & 0.9734 & 0.7701 & 0.7735 & 0.7898 & 0.7802 & 0.9750 & 0.8117 & 0.7993 \\
\hline
\end{tabular}

Table III. EXECUTIVE Times OF THE GPU-BASED IMPLEMENTATION (IN SECOND).

\begin{tabular}{|c|c|c|c|c|c|c|c|c|c|c|}
\hline \multirow{2}{*}{\multicolumn{2}{|c|}{ Methods }} & \multirow{3}{*}{$\begin{array}{c}\text { Workspace } \\
\mathrm{CPU}\end{array}$} & \multicolumn{3}{|c|}{ Fig. 6} & \multicolumn{3}{|c|}{ Fig. 7} & \multicolumn{2}{|c|}{ Fig. 8} \\
\hline & & & (a) & (b) & (c) & (a) & (b) & (c) & (a) & (b) \\
\hline \multirow{3}{*}{ A-DLSO } & K-means & & 0.6468 & 0.4394 & 0.4366 & 0.4706 & 0.4631 & 0.6523 & 0.5062 & 0.4733 \\
\hline & $\begin{array}{c}\text { Curve } \\
\text { evolution }\end{array}$ & GPU & 0.0204 & 0.0198 & 0.0193 & 0.0199 & 0.0202 & 0.0205 & 0.0201 & 0.0198 \\
\hline & Total & & 0.6672 & 0.4592 & 0.4559 & 0.4905 & 0.4833 & 0.6728 & 0.5263 & 0.4931 \\
\hline \multicolumn{2}{|c|}{$\mathrm{CV}$} & \multirow[b]{2}{*}{ GPU } & 47.004 & 46.751 & 47.722 & 46.768 & 46.659 & 47.604 & 62.573 & 46.764 \\
\hline \multicolumn{2}{|c|}{ HZ } & & 0.04282 & 0.04334 & 0.04358 & 0.04360 & 0.04363 & 0.04318 & 0.04312 & 0.04338 \\
\hline
\end{tabular}




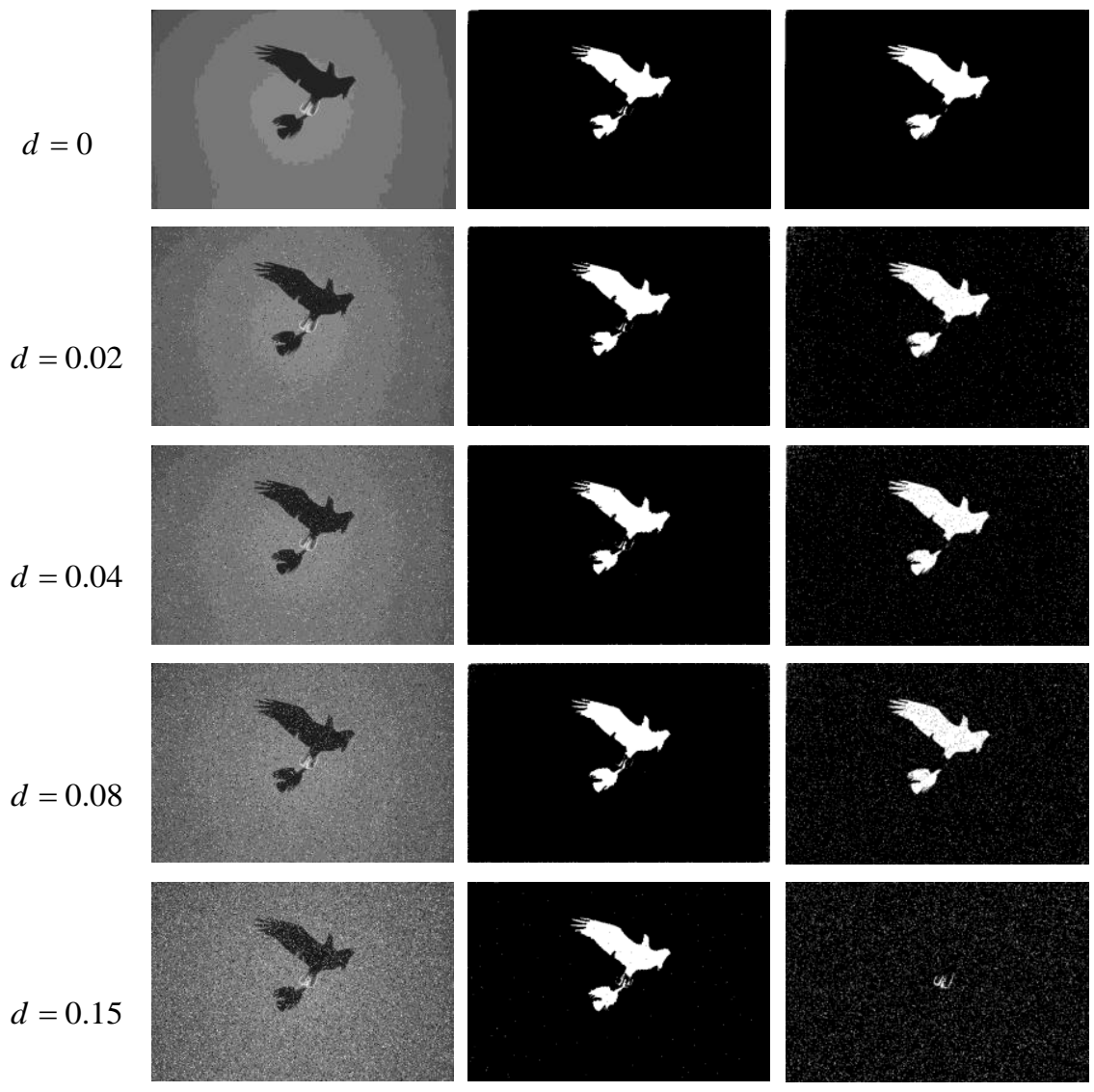

Fig. 5. Segmentation of an image corrupted by salt and pepper noise with different density values $d$. The first column displays the corrupted images. The second column presents the results of the proposed method. The third column presents the results of the k-means algorithm.

[7] S. Balla-Arabé, X.-B Gao, B. Wang, F. Yang and V. Brost, "Multi-Kernel Implicit Curve Evolution for Selected Texture Regions Segmentation in VHR Satellite Images," IEEE Transactions on Geoscience and Remote Sensing, vol. 52, no. 8, pp. 5183-5192, 2014.

[8] Y. Boycov, O. Veksler, and R. Zabih, "Fast Approximate Energy Minimization Via Graph Cuts," IEEE Transactions on Pattern Analysis and Machine Intelligence, vol. 23, no. 11, pp. 1222-1239, 2001.

[9] J. Malik, S. Belongie, T. Leung, and J. Shi, "Contour and Texture Analysis for Image Segmentation," International Journal of Computer Vision, 43(1):7-27, 2001.

[10] G. Papandreou, and P. Maragos, "Multigrid Geometric Active Contour Models," IEEE Transactions on Image Processing, vol. 16, no. 1, pp. 229-240, 2007.

[11] B. Wang, X.-B Gao, D. Tao and X. Li, “A Nonlinear Adaptive Level Set for Image Segmentation," IEEE Transactions on Cybernetics, vol. 44, no. 3, pp. 418-428, 2014.

[12] C. Li, C. Kao, J. Gore, and Z. Ding, "Implicit Active Contours Driven by Local Binary Fitting Energy," Proceedings of IEEE Conference in Computer Vision and Pattern Recognition, pp. 1-7, 2007.

[13] C. Li, R. Huang, Z. Ding, J. Chris, D.N. Metaxas, and J.C. Gore, “A Level Set Method for Image Segmentation in the Presence of Intensity Inhomogeneities With Application to MRI," IEEE Transactions on Image Processing, vol. 20, no. 7, pp. 2007-2016, July 2011.

[14] S. Zhu, and A. Yuille, "Region Competition: Unifying Snakes, Region Growing, and Bayes/MDL for Multiband Image Segmentation," IEEE Transactions on Pattern Analysis and Machine Intelligence, vol. 18, no. 9, pp. 884-900, 1996.

[15] C. Brun, N. Leporé, X. Pennec, Y. Chou, A. Lee et al., "A Nonconservative Lagrangian Framework for Statistical Fluid
Registration-SAFIRA," IEEE Transactions on Medical Imaging, vol. 30, no. 2, pp. 184-202, 2011.

[16] A. Nakhmani, and A. Tannenbaum, "Self-crossing Detection and Location for Parametric active contours," IEEE Transactions on Image Processing, vol. 21, no. 7, pp. 3150-3156, 2012.

[17] M. Kass, A. Witkin, and D. Terzopoulos, "Snakes: Active Contour Models," International Journal of Computer Vision, vol. 1, no.4, pp. 321-331, 1988.

[18] S. Osher, and J. Sethian, "Fronts Propagating and Curvature Dependent Speed: Algorithms Based on Hamilton-Jacobi Formulation," J. Comput. Phys., 79(1):12-49, 1988.

[19] R. Malladi, J. Sethian, and B. Vemuri, "A Topology Independent Shape Modeling Scheme," Proceedings of SPIE Conference on Geometric Methods in Computer Vision II, vol. 2031, pp. 246-258, San Diego, CA, 1993.

[20] V. Caselles, R. Kimmel, and G. Sapiro, "On Geodesic Active Contours," International Journal of Computer Vision, 22(1):61-79, 1997.

[21] X-B. Gao, B. Wang, D. Tao, and X. Li, "A Relay Level Set Method for Automatic Image Segmentation," IEEE Transactions on Systems, Man and Cybernetics Part B: Cybernetics, 41(2):518-525, April 2011.

[22] S. Kichenassamy, A. Kumar, P. Olver, A. Tannenbaum, and A. Yezzi, "Gradient Flows and Geometric Active Contours Models," Proceedings of the 5th International Conference on Computer Vision, pp. 810-8155, June 1995.

[23] D. Cremers, M. Rousson, and R. Deriche, "A Review of Statistical Approaches to Level Set Segmentation: Integrating Color, Texture, Motion and Shape," International Journal of Computer Vision, 72(2):195-215, 2007.

[24] R. Delgado-Gonzalo, P. Thévenaz, C.S. Seelamantula, and M. Unser, 
"Snakes with an Ellipse-Reproducing Property," IEEE Transactions on Image Processing, vol. 21, no. 3, pp. 1258-1271, March 2012.

[25] C. Li, C. Xu, C. Gui, and M.Fox, "Distance Regularized Level Set Evolution and its Application to Image Segmentation," IEEE Transactions on Image Processing, vol. 19, no. 12, pp. 3243-3254, December 2010.

[26] S. Balla-Arabé, X.-B Gao, and B. Wang, "GPU Accelerated Edge-Region Based Level Set Evolution Constrained By 2D Gray-scale Histogram,"
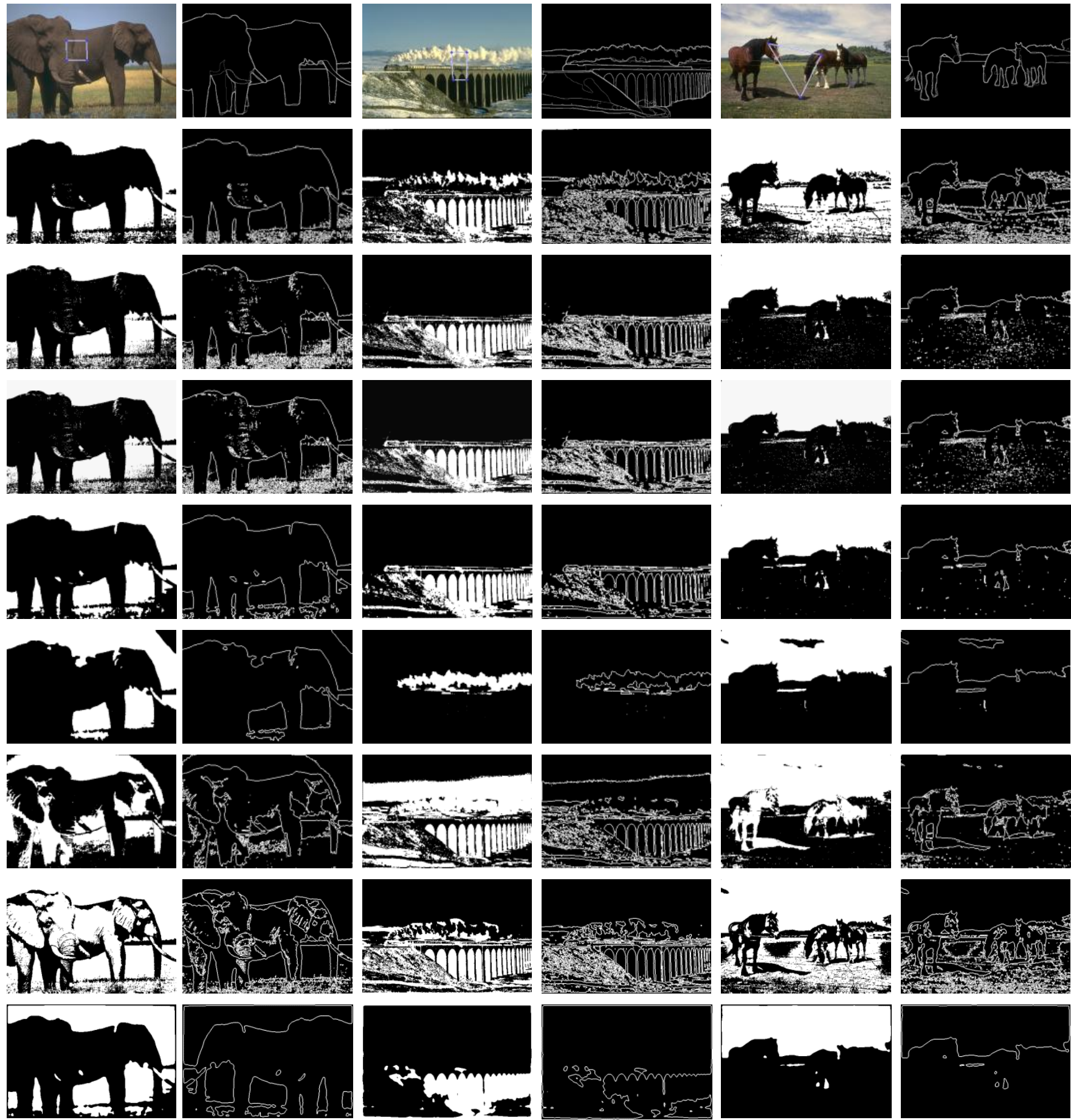

(a)
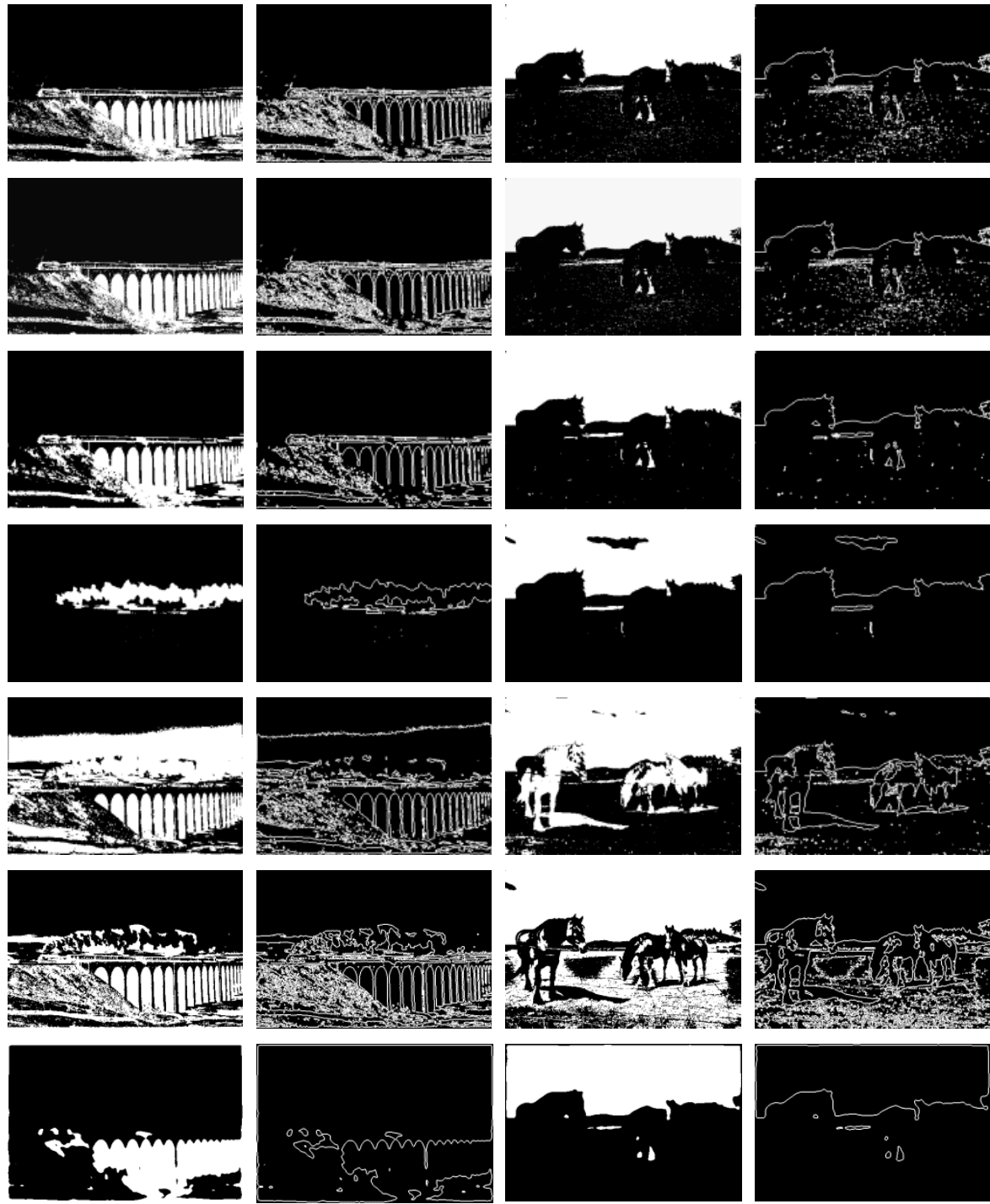

(b)

(c)

Fig. 6. Segmentation of real-world images. The first row presents the original images with the initial contour, and the corresponding human segmentation as ground truth. The second row presents the results of the proposed algorithm. The third row presents the results of the k-means clustering method. The fourth row presents the results of the FCM clustering method. The fifth row presents the results of the PKGC method. The sixth row presents the results of the CV method. The seventh row presents the results of the HZ method. The eighth row presents the results of the Li method. The last row presents the results of the GF method. For all the methods, the results are presented in two ways, i.e., by contours and binary images. 
[28] S. Balla-Arabé, B. Wang, and X-B. Gao, "Level Set Region Based Image Segmentation Using Lattice Boltzmann Method," Proceedings of the 7th International Conference on Computational Intelligence and Security, pp. 1159-1163, Sanya, China, December 2011.

[29] T. Chan, and L. Vese, "Active Contours Without Edges," IEEE Transactions on Image Processing, vol.10, no.2, pp. 266-277, 2001.

[30] D. Mumford, and J. Shah, "Optimal Approximations by Piecewise Smooth Functions and Associated Variational Problems," Commun. Pure
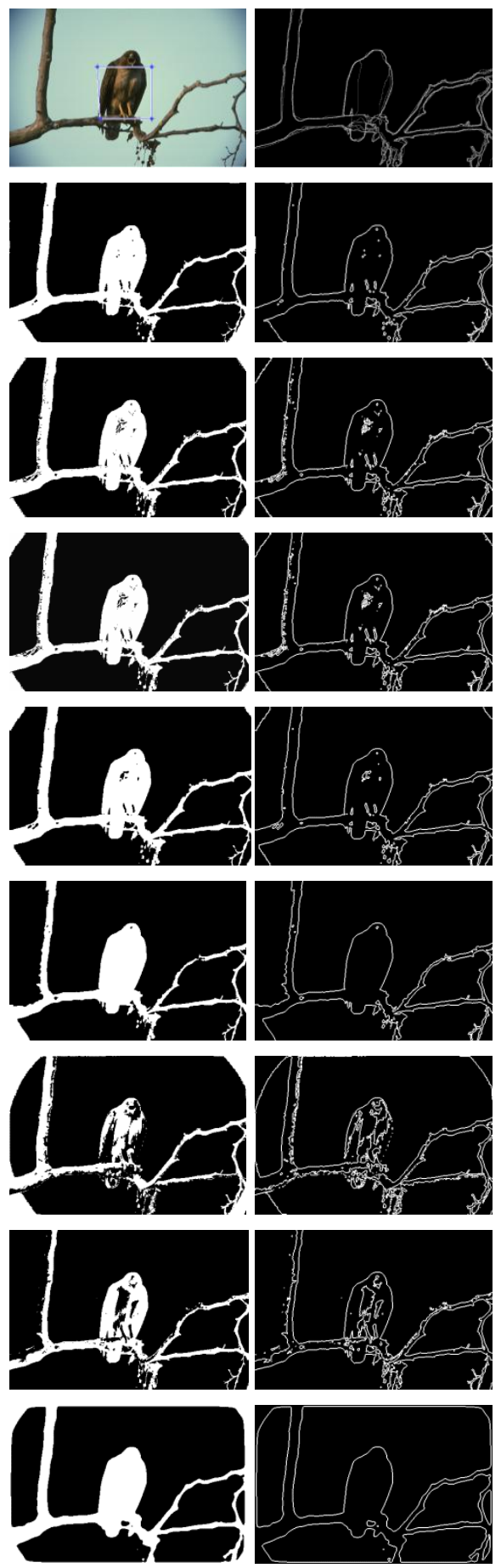

(a)
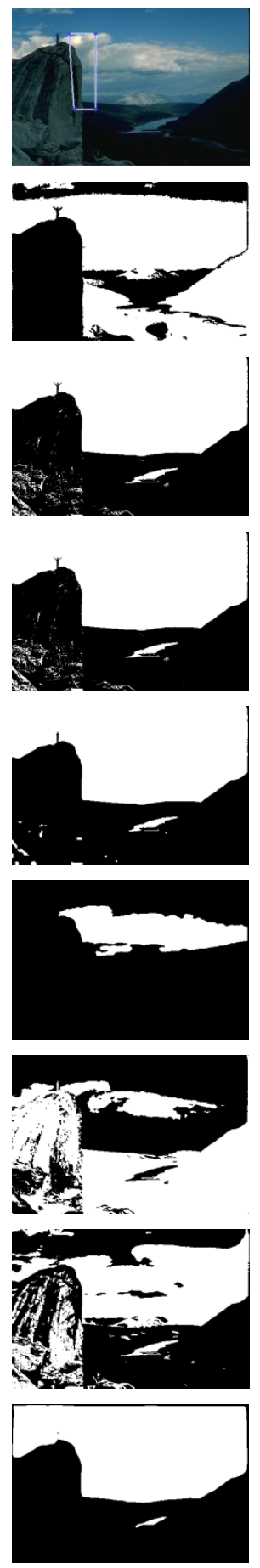

(b)
Appl. Math., 42(5):577-685, 1989.

[31] S. Osher, and R. Fedkiw, Level set methods and dynamic implicit surfaces, Springer, 2003.

[32] L. Vese, and T. Chan, "A Multiphase Level Set Framework for Image Segmentation Using the Mumford and Shah Model," International Journal of Computer Vision, vol. 50, no. 3, pp. 271-293, 2002.
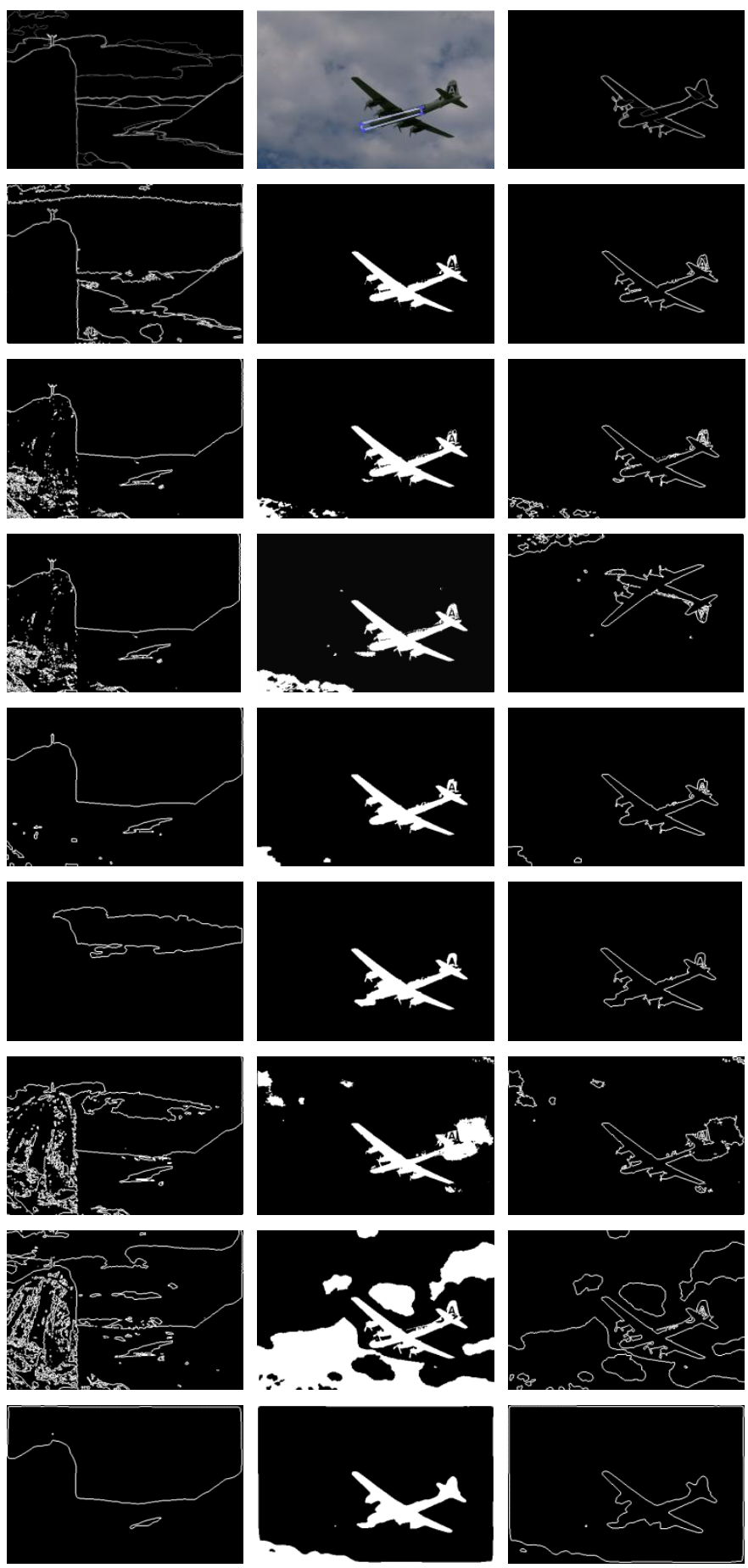

(c)

Fig. 7. Segmentation of real-world images. The first row presents the original images with the initial contour, and the corresponding human segmentation as ground truth. The second row presents the results of the proposed algorithm. The third row presents the results of the k-means clustering method. The fourth row presents the results of the FCM clustering method. The fifth row presents the results of the PKGC method. The sixth row presents the results of the CV method. The seventh row presents the results of the HZ method. The eighth row presents the results of the Li method. The last row presents the results of the GF method. For all the methods, the results are presented in two ways, i.e., by contours and binary images. 
[33] Y. Chen, H. Tagare, S. Thiruvenkadam, F. Huang, D. Wilson, K. Gopinath, R. Briggs, and E. Geiser, "Using prior shapes in geometric active contours in a variational framework," International Journal of Computer Vision, 50(3):315-328, 2002.

[34] C. Li, C. Kao, J. Gore, and Z. Ding, "Minimization of Region-Scalable Fitting Energy for Image Segmentation," IEEE Transactions on Image Processing, vol. 17, no. 10, pp. 1940-1949, 2008.

[35] D. Martin, C. Fowlkes, D. Tal, and J. Malik, “A Database of Human segmented Natural Images and its Application to Evaluating Segmentation Algorithms and Measuring Ecological Statistics," Proceedings of 8th International Conference on Computer Vision, vol. 2, pp. 416-423, 2001.

[36] F. Gibou, and R. Fedkiw, "A Fast Hybrid k-means Level Set Algorithm for Segmentation," Proceedings of the 4th Annual Hawaii International Conference on Statistics and Mathematics, pp. 281-291, 2005.
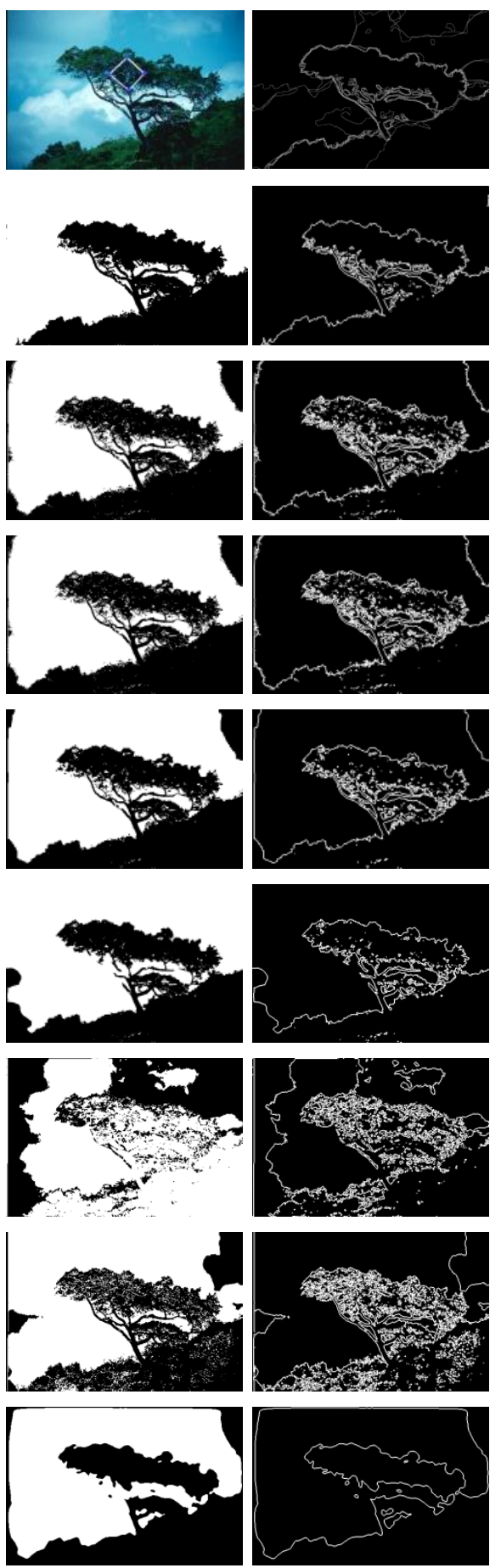

(a)
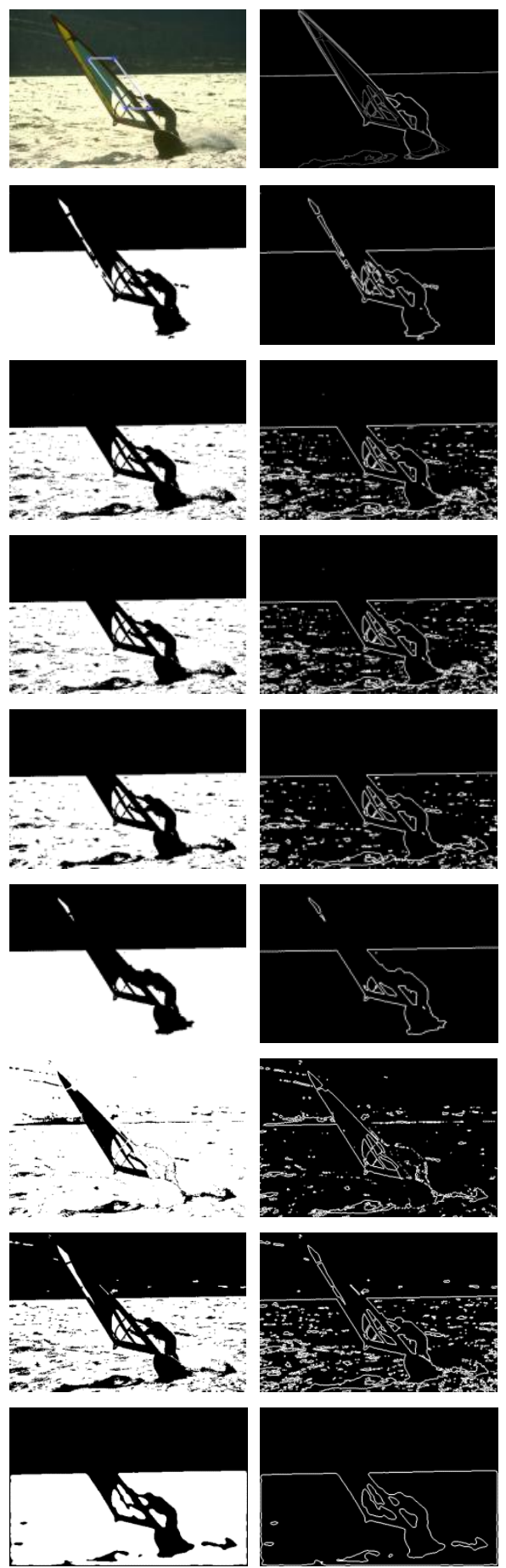

(b)

Fig. 8. Segmentation of real-world images. The first row presents the original images with the initial contour, and the corresponding human segmentation as ground truth. The second row presents the results of the proposed algorithm. The third row presents the results of the k-means clustering method. The fourth row presents the results of the FCM clustering method. The fifth row presents the results of the PKGC method. The sixth row presents the results of the CV method. The seventh row presents the results of the HZ method. The eighth row presents the results of the Li method. The last row presents the results of the GF method. For all the methods, the results are presented in two ways, i.e., by contours and binary images. 


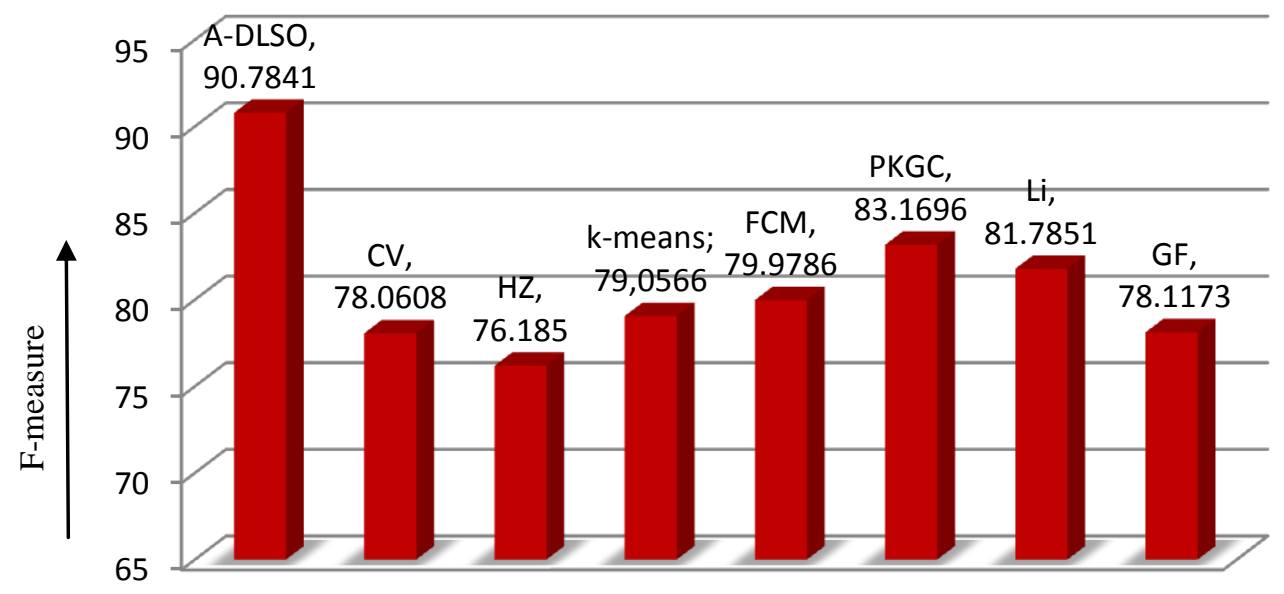

Fig. 9. 3D histogram showing the supervised objective evaluation. The vertical axis represents the F-measure, while the horizontal axis represents the evaluated methods.

(a)

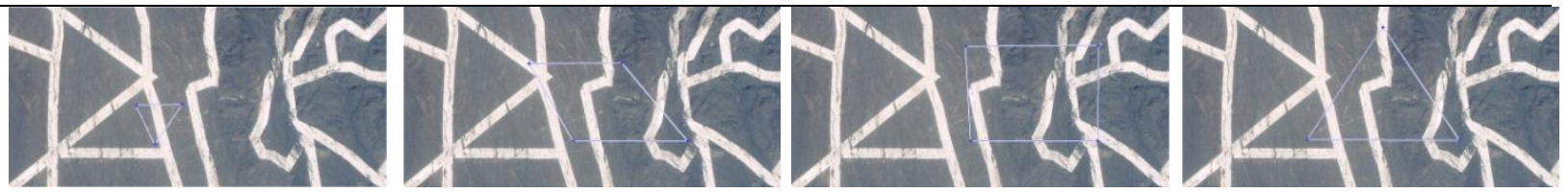

(b)



(c)
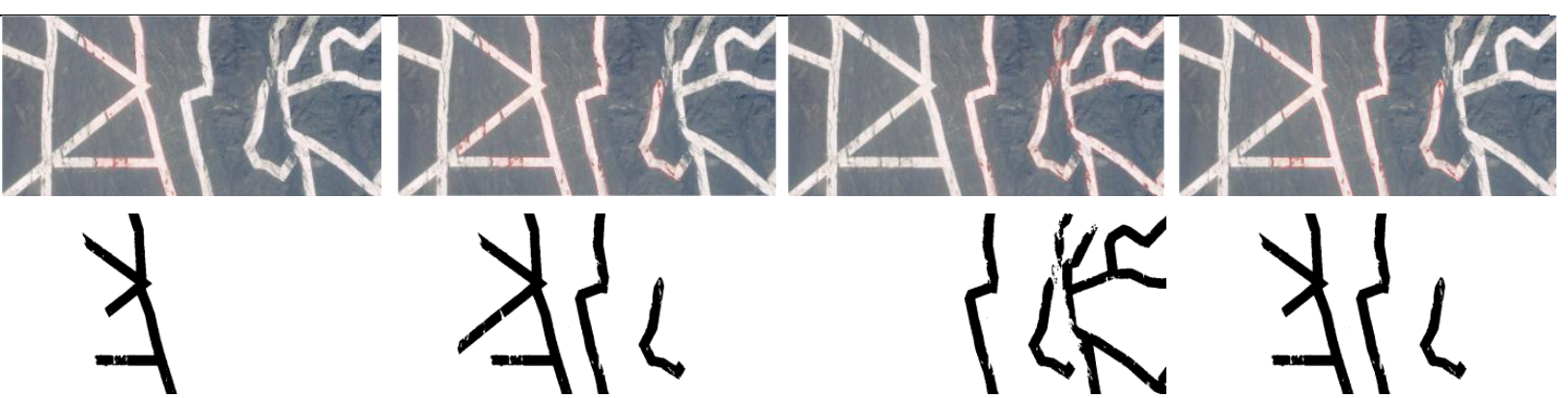

Fig. 10. Segmentation of a satellite image of Xinjiang in China with different initial contours. The results are presented in two ways, i.e., an image where the resulting contour is held on the original image, and a binary image which represents the interior of the final LSF by white pixels and the exterior by black pixels. (a) Initial Contours. (b) Results of the proposed method. (c) Results of the CV method.

[37] S. Succi, The Lattice Boltzmann Equation for Fluid Dynamics and Beyond Numerical Mathematics and Scientific Computation, Oxford University Press, New York, 2001.

[38] Ye Zhao, "Lattice Boltzmann Based PDE Solver on the GPU," The Visual Computer, vol. 24, no. 5, pp. 323-333, Springer, May 2007.

[39] X. He, and L. Luo, "Lattice Boltzmann Model for Incompressible Navier-Stokes Equation,” J. Stat. Phys., vol. 88, no. 3-4, pp. 927-944, 1997.

[40] P.L. Bhatnagar, E.P. Gross, and M. Krook, "A Model for Collision Processes in Gases. I. Small Amplitude Processes in Charged and Neutral One-component Systems," Phys. Rev., vol. 94, no. 3, pp. 511-525, 1954.
[41] J. Buick, and C. Greated, "Gravity in a lattice Boltzmann model," Phys. Rev. E, vol.61(5), pp. 5307-5320, 2000.

[42] X. Shan, and G. Doolen, J. Stat. Phys., vol. 81, 379, 1995.

[43] I. Ginzbourg, and P.M. Adler, J. Phys. II, vol. 4, 191, 1994.

[44] S. Chapmann, and T.G. Cowling, The Mathematical Theory of Non-Uniform Gases: An account of the kinetic Theory of Viscosity, Thermal Conduction and Diffusion in Gases, Cambridge University Press, Cambridge, U.K., 1990.

[45] Y. Chen, Z. Yan, and Y. Chu, "Cellular Automata Based Level Set Method for Image Segmentation," IEEE/ICME International Conference on Complex Medical Engineering, Beijing, pp. 23-27, May 2007. 
[46] G. Aubert, and P. Kornprobst, "Mathematical Problems in Image Processing: Partial Differential Equations and the Calculus of Variations," Applied Mathematical Sciences, vol. 147, Springer-Verlag, 2001.

[47] J. G. Rosen, "The Gradient Projection Method for Nonlinear Programming, II, Non-linear Constraints," J. SIAM, vol.9, pp. 514-532, 1961.

[48] S. Wang, and X. Yao, "Relationships Between Diversity of Classification Ensembles and Single-class Performance Measures," IEEE Transactions on knowledge and data engineering, vol. 25, no. 1, pp. 206-219, 2013.

[49] H. He, and E. A. Garcia, "Learning from Imbalanced Data," IEEE Transactions on knowledge and data engineering, vol. 21, no. 9, pp. 1263-1284, 2009.

[50] M. Ben Salah, A. Mitiche, and I. Ben Ayed, "Multiregion Image Segmentation by Parametric Kernel Graph Cuts," IEEE Transactions on Image Processing, vol. 20, no. 2, pp. 545-557, 2011.

[51] A. Hagan, and Y. Zhao, "Parallel 3D Image Segmentation of Large Data Set on a GPU Cluster," in Proceedings of the 5th International Symposum on Visual Computing, pp. 960-969, Springer, 2009.

[52] Y. Bazi, F. Melgani, and H. Al-Sharari, "Unsupervised Change Detection in Multispectral Remotely Sensed Imagery with Level set Methods," IEEE Transactions and Geoscience and Remote Sensing, vol. 48, no. 8, pp. 3178-3187, Aug. 2010.

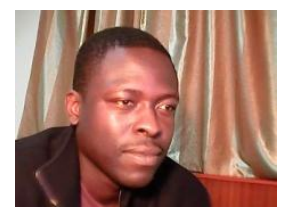

Souleymane Balla-Arabé (M'12) received the M.Eng. degree in electronic from Polytechnic Military School, Algiers, Algeria, in 2004, and the $\mathrm{Ph} . \mathrm{D}$. degree in information and communication engineering at Xidian University, Xi'an, China in 2014. He is currently a research fellow at LE2I CNRS-UMR, Laboratory of Electronic, Computing, and Image Sciences, University of Burgundy, France. His research interests are architecture-aware optimization strategies for image segmentation, GPU-based computations, computational intelligence, machine learning, and computer vision. In these areas, he has published several technical articles in refereed journals and proceedings including IEEE TRANSACTIONS ON IMAGE PROCESSING, the IEEE TRANSACTIONS ON GEOSCIENCE AND REMOTE SENSING, and the IEEE TRANSACTIONS ON CYBERNETICS.

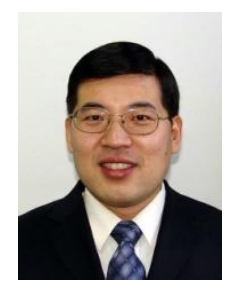

Xinbo Gao (M'02-SM'07) received the B.Eng., M.Sc., and $\mathrm{Ph} . \mathrm{D}$. degrees in signal and information processing from Xidian University, Xi'an, China, in 1994, 1997, and 1999, respectively. From 1997 to 1998, he was a Research Fellow at the Department of Computer Science, Shizuoka University, Shizuoka, Japan. From 2000 to 2001, he was a Post-Doctoral Research Fellow at the Department of Information Engineering, the Chinese University of Hong Kong, Hong Kong. Since 2001, he has been at the School of Electronic Engineering, Xidian University. He is currently a Cheung Kong Professor of Ministry of Education, a Professor of Pattern Recognition and Intelligent System, and the Director of the State Key Laboratory of Integrated Services Networks, Xi'an, China. His current research interests include multimedia analysis, computer vision, pattern recognition, machine learning, and wireless communications. He has published five books and around 200 technical articles in refereed journals and proceedings. Prof. Gao is on the Editorial Boards of several journals, including Signal Processing (Elsevier), and Neurocomputing (Elsevier). He served as the General Chair/ Co-Chair, Program Committee Chair/Co-Chair, or PC Member for around 30 major international conferences. He is currently a fellow of the Institution of Engineering and Technology.

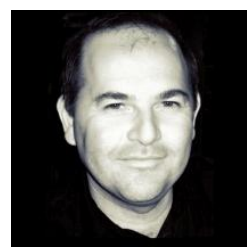

Dominique Ginhac received his Master's Degree in Engineering (1995) followed by a Ph.D in Computer Vision (1999) from the Blaise Pascal University (France). He then joined the University of Burgundy as an assistant professor (2000) and became member of Le2i UMR CNRS 6306 (Laboratory of Electronic, Computing and Imaging Sciences). In 2009, he was promoted Professor and became head of the electrical engineering department until 2011 . He is currently deputy director of the Le2i laboratory. His research activities were first in the field of rapid prototyping of real-time image processing on dedicated parallel architectures. More recently, he has developed an expertise in the field of image acquisition, hardware design of smart vision systems and implementation of real-time image processing applications.

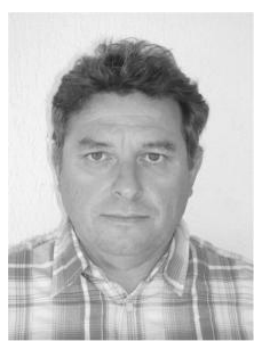

Vincent BROST received the Master Degree and Ph.D degrees from University of Burgundy, in 2000 and 2006, respectively. He is currently an associate professor at the University of Burgundy and member of LE2I (Laboratory of Electronic, Computing, and Imaging), France. His research interests are in the areas of rapid prototyping system, VLIW architecture, and Electronic Devices.

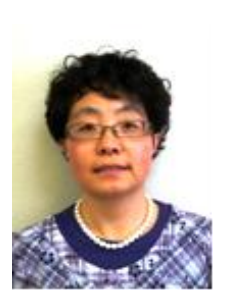

Fan Yang was born in Tianjin, China in 1963. She received the B.S. degree in electrical engineering from University of Lanzhou, China in 1982. She received the M.S. degree in computer science and Ph.D. degree in image processing from University of Burgundy, France, in 1994 and 1998, respectively. She is currently a full professor and member of LE2I CNRS-UMR, Laboratory of Electronic, Computing, and Image Sciences at the University of Burgundy, France. Her research interests are in the areas of patterns recognition, neural network, motion estimation based on spatial-temporal Gabor filters, parallelism and real-time implementation, and, more specifically, biometric image processing algorithms and architectures. Prof. Yang is member of the French research group ISIS (Information, Signal, Images and Vision), she livens up the theme C: Algorithm Architecture Mapping. 